\title{
Honokiol Protects against Anti- $\beta 1$-Adrenergic Receptor Autoantibody-Induced Myocardial Dysfunction via Activation of Autophagy
}

\author{
Xi-qing Wei, ${ }^{1}$ Hong-sheng Zhang, ${ }^{1}$ Guang-he Wei, ${ }^{1}$ Jin-guo Zhang, ${ }^{1}$ Yan-yan Du, \\ Hong-yong Tan, ${ }^{1}$ and Jun Yang $\mathbb{1}^{2,3}$ \\ ${ }^{1}$ Department of Cardiology, Affiliated Hospital of Jining Medical University, Jining, Shandong 272129, China \\ ${ }^{2}$ Department of Cardiology, Yantai Yuhuangding Hospital, Affiliated Hospital of Qingdao University, Yantai, \\ Shandong 264000, China \\ ${ }^{3}$ Qingdao University, Qingdao, Shandong 266071, China
}

Correspondence should be addressed to Jun Yang; bioyangjun@126.com

Received 7 February 2018; Revised 4 May 2018; Accepted 30 May 2018; Published 18 July 2018

Academic Editor: Silvana Hrelia

Copyright (C) $2018 \mathrm{Xi}$-qing Wei et al. This is an open access article distributed under the Creative Commons Attribution License, which permits unrestricted use, distribution, and reproduction in any medium, provided the original work is properly cited.

\begin{abstract}
Myocardial diseases are prevalent syndromes with high mortality rate. The exploration of effective interference is important. Anti$\beta 1$-adrenergic receptor autoantibody $(\beta 1-\mathrm{AAB})$ is highly correlated with myocardial dysfunction. The actions and underlying mechanisms of honokiol (HNK) in $\beta 1$-AAB-positive patients await to be unraveled. In this study, we established a rat model of $\beta 1-\mathrm{AAB}$ positive with myocardial dysfunction. Cardiac function following $\beta 1$-AR-ECII administration was analyzed using the VisualSonics Vevo 770 High-Resolution In Vivo Imaging System. The levels of autophagy-related proteins were detected by Western blotting. Our data revealed that HNK reversed $\beta 1$-AAB-induced effects and protected myocardial tissues from dysfunction. After HNK treatment, the cardiac contractile ability increased and the LDH activity decreased. HNK attenuated myocardial degeneration. In addition, HNK promoted the activation of the AMP-dependent protein kinase/Unc-51-like autophagy activating kinase (AMPK/ULK) pathway and activated autophagy. These results suggest that HNK protects against $\beta 1-\mathrm{AAB}$-induced myocardial dysfunction via activation of autophagy and it may be a potentially therapeutic compound for $\beta 1$-AAB-positive myocardial diseases.
\end{abstract}

\section{Introduction}

Myocardial dysfunction is a complicated syndrome characterized by reduced ejection fraction, biventricular dilatation, and decreased response to fluid administration [1]. Multiple factors, such as sepsis and ischemia/reperfusion, can induce myocardial dysfunction. Cardiovascular abnormalities significantly contribute to the mortality of septic patients [2]. In China, the prevalence of cardiovascular diseases shows an upward trend [3]. Despite the progress in cardiac disease treatment, the mortality rate is still high [4]. Among these mortalities, myocardial dysfunction accounts for approximately $40 \%$ [5]. Further exploration of effective intervention for myocardial dysfunction is the key to improving the survival and recovery of patients with cardiac dysfunction.

It has been shown that anti- $\beta 1$-adrenergic receptor autoantibodies ( $\beta 1-\mathrm{AAB})$ are significantly distributed in the sera of patients with dilated cardiomyopathy and ischemic cardiomyopathy [6,7]. Binding of specific autoantibodies can lead to increased pulsatility and atrial contractility and activation of the $\mathrm{Ca}^{2+}$ channels [8]. Previous studies have demonstrated that the long-term existence of $\beta 1-\mathrm{AAB}$ causes cardiac function impairments [6,9]. Cardiomyocyte death is one of the major factors causing heart dysfunction [10]. $\beta 1$ $\mathrm{AAB}$ induces the death of adult rat ventricular myocytes in vitro, which may be related to cardiomyocyte apoptosis 
[11]. It has been shown that $\beta 1-\mathrm{AAB}$ and its agonist isoproterenol decrease autophagy and are critical for the maintenance of cardiomyocyte homeostasis [12]. Thus, $\beta 1-\mathrm{AAB}$ can be used to establish a rat model of myocardial dysfunction.

Honokiol (HNK) is a biphenolic compound with antioxidative, anti-inflammatory, and neuroprotective properties [13]. Oral treatment of HNK ameliorates age-related learning and memory dysfunction [14]. In rats, HNK protects the cortex and heart from ischemia/reperfusion injury via inhibition of reactive oxygen species production [15]. HNK induces apoptosis of various tumor cells and reverses epithelialmesenchymal transition induced by $\operatorname{TGF} \beta$ and TNF $\alpha$ [13]. Early studies have demonstrated that in rats with myocardial infarction, HNK administration limits infarct size and shows antiarrhythmic effects [16]. These findings suggest that HNK has cytoprotective capabilities. Although HNK has been used to treat various vascular diseases, such as ischemia and stroke [17], whether HNK is beneficial in $\beta 1$-AAB-positive heart diseases is unclear and the underlying mechanisms remain to be explored.

The aim of this study is to investigate the effects of HNK on myocardial injury in a rat model of $\beta 1$-AAB-positive in vivo and to reveal its mechanisms.

\section{Materials and Methods}

2.1. Establishment of a Rat Model of $\beta 1-A A B$-Positive and HNK Treatment. Healthy adult male Wistar rats (8 weeks) were obtained from the animal center. Rats were randomly divided into four groups: control group, $\beta 1-\mathrm{AAB}$ group, $\beta 1-\mathrm{AAB}+\mathrm{HNK}$ group, and $\beta 1-\mathrm{AAB}+$ vehicle group. $\beta 1-$ AR-ECII [a synthetic peptide with $100 \%$ rat homology (197 223, H-W-W-R-A-E-S-D-E-A-R-R-C-Y-N-D-P-K-CC-D-F-V-T-N-R-A, synthesized by GL Biochemical Co., LTD, Shanghai)] was dissolved using $\mathrm{Na}_{2} \mathrm{CO}_{3}$ solution $(1 \mathrm{mg} / \mathrm{ml})$ and emulsified using complete Freund's adjuvant (CFA) (1:1, Sigma-Aldrich, F5881) [18]. Rats in the $\beta 1-\mathrm{AAB}$ group were subcutaneously injected with this emulsified antigen $(0.4 \mu \mathrm{g} / \mathrm{g})$. We first injected multiple sites posteriorly along the back of rats. After that, a booster immunization $(0.4 \mu \mathrm{g} / \mathrm{g})$ was given every two weeks. Rats in the control group were injected with normal saline in CFA in the same manner. Each rat blood was collected one day before booster injection, and the formation of $\beta 1-\mathrm{AAB}$ was detected. Rat sera were extracted using MAbTrap Kit (GE Healthcare, 17-1128-01, Uppsala, Sweden) for the purification of IgG and then analyzed by an ELISA kit. Honokiol was purchased from Sigma-Aldrich (St. Louis, MO) and prepared as described previously [19].

2.2. Echocardiography Examinations. We examined cardiac function at $12 \mathrm{~h}$ after $\beta 1$-AR-ECII administration by using the VisualSonics Vevo 770 High-Resolution In Vivo Imaging System (VisualSonics Inc., Toronto, Ontario, Canada). The Doppler and M-mode echocardiography was performed according to previous studies [20]. Rats were anesthetized by a mixed gas of oxygen and $2 \%$ isoflurane (Rhodia UK Ltd., Avonmouth, Bristol, UK). We performed echocardiography with a $30 \mathrm{MHz}$ center frequency RMV 707 scan head
(VisualSonics Inc.). All the echocardiography examinations were performed by the same technician. All the results were from three cardiac cycles.

2.3. Measurements of Cardiac Function. As previously described [21], a cannula was inserted into the left ventricle via the right carotid artery. BL-410 recording and analysis system was applied to record and analyze the following parameters: the left ventricular systolic pressure (LVSP), left ventricular end diastolic pressure (LVEDP), and maximal positive and negative values of the instantaneous first derivative of left ventricular pressure $\left(+\mathrm{dP} / \mathrm{dt}_{\max }\right.$ and $\left.-\mathrm{dP} / \mathrm{dt}_{\max }\right)$.

2.4. Measurement of Lactate Dehydrogenase (LDH). Two hundred thousand cells were plated at the 6-well plates overnight. The culture medium was collected for $\mathrm{LDH}$ assays. LDH was measured using a LDH assay kit (Abcam, ab65393). The data were normalized according to the number of cells.

2.5. HE Staining. Cardiac tissues were rinsed with PBS and then fixed by $4 \%$ paraformaldehyde. The tissues were dehydrated with $30 \%$ sucrose in PBS overnight at $4{ }^{\circ} \mathrm{C}$ and then embedded using embedding medium. Sections were obtained using a freezing microtome. Slides were put into xylene and $100 \%$ ethanol in order (each three times, $3 \mathrm{~min} /$ time) and then into $95 \%$ ethanol (one time, $3 \mathrm{~min}$ ), $80 \%$ ethanol (one time, $3 \mathrm{~min}$ ) and deionized water (one time, $5 \mathrm{~min}$ ) in turn. After that, the sections were immersed in hematoxylin (one time, $3 \mathrm{~min}$ ), rinsed with deionized water and tap water in order (each one time, $5 \mathrm{~min}$ ). Acid ethanol was used to destain the slides. After rinsing with tap water (two times, $1 \mathrm{~min}$ ) and deionized water (one time, $2 \mathrm{~min}$ ), sections were immersed in eosin (one time, 30 seconds). After rinsing with 95\% and $100 \%$ ethanol (each three times, $5 \mathrm{~min} /$ time), slides were put into xylene (three times, $15 \mathrm{~min} /$ time). At last, slides were mounted with permount.

2.6. Immunofluorescence. Cultured H9c2 cells were exposed to $40 \mu \mathrm{M}$ honokiol for $72 \mathrm{~h}$ and were washed twice using PBS. After that, cells were fixed by $4 \%$ paraformaldehyde for $15 \mathrm{~min}$ and then washed using PBS. Next, cells were incubated using $0.2 \%$ Triton in PBS for 0.5 hours. After that, cells were blocked by blocking buffer for $1 \mathrm{~h}$ at room temperature. Last, cells were incubated with LC3B antibody $(1: 400$; Cell Signaling, 2775) in a humidified box overnight at $4^{\circ} \mathrm{C}$. On the second day, cells were first washed using PBS and then incubated with a second antibody for $2 \mathrm{~h}$. After washing with PBS, we added 2-(4-amidino-phenyl)-6-indolecarba-midine dihydrochloride (DAPI, Beyotime Biotech, C1005) solution to stain the nuclei for $3 \mathrm{~min}$. Cells were washed using PBS and then sealed with a coverslip. The images were captured using laser confocal microscopy (OLYMPUS, FV1000, USA).

2.7. Antibodies and Reagents. LC3 and p62 antibodies were purchased from Novus Biologicals (Abcam, ab48394) and Enzo Life Sciences (Abcam, ab56416), respectively. Other antibodies were obtained from Cell Signaling (Boston, MA, USA). 
2.8. Western Blot. Extracts from tissues and cultured cells were prepared as previously described [22]. The homogenates were centrifuged $(14,000 \mathrm{rpm}, 30 \mathrm{~min})$ at $4^{\circ} \mathrm{C}$, and the supernatants were collected. We determined protein concentrations using the BCA Protein Assay (Thermo Scientific, Rockford, IL). Protein lysates (approximately $40 \mu \mathrm{g}$ ) were separately loaded on $10 \%$ polyacrylamide gels and then transferred onto polyvinylidene difluoride membranes. The membranes were blocked by blocking buffer (5\% milk in PBS) for $30 \mathrm{~min}$ at room temperature. After that, the primary antibodies $(1: 10,000)$ were added and incubated overnight at $4^{\circ} \mathrm{C}$. The membranes were washed in PBST (PBS with $0.2 \%$ Tween-20) and incubated with secondary antibodies $(1: 10,000)$ for $1 \mathrm{~h}$ at room temperature. The membranes were washed in PBST, and the images were captured using the Odyssey Infrared Imaging System (LiCOR Biotechnology).

2.9. Statistical Analysis. Data were shown as the mean \pm standard deviation (SD). We performed the statistical analysis by SPSS software (version 15.0, SPSS Inc., Chicago, IL, USA). We used Student's $t$-test to compare the means of two independent groups and applied one-way ANOVA with a Bonferroni post hoc test for more than two independent samples. Statistical significance was defined when $P<0.05$.

\section{Results}

3.1. A Rat Model of Long-Term Maintenance of $\beta 1-A A B$ Was Established. To establish a rat model of long-term maintenance of $\beta 1$-AAB, $\beta 1$-AR-ECII or saline were subcutaneously injected into adult rats. $\beta 1-\mathrm{AAB}$ titer in the blood was then defined by $\mathrm{OD}$ value. At the beginning of immunization, the protein levels of $\beta 1$-AABs in the sera were both very low in the two groups (Figure 1). Compared to the saline treatment group, $\beta 1$-AABs were sharply increased in the first four weeks and maintain a high level later in the $\beta 1$-AR-ECII treatment group (Figure 1). These data indicate that we have successfully established a rat model of long-term high-level maintenance of $\beta 1-\mathrm{AAB}$.

\subsection{HNK Prevented $\beta 1-A A B$-Provoked Myocardial} Dysfunction. To investigate the cardiac ventricular function, echocardiography was used to examine it at $12 \mathrm{~h}$ following injection with saline or $\beta 1$-AR-ECII [23]. As shown in Figure 2, the M-mode echocardiography evaluation showed that compared to the control group, significant suppression of cardiac ventricular contractility was observed in the group with $\beta 1$-AR-ECII treatment (Figures $2(\mathrm{a})$ and $2(\mathrm{~b})$ ). In contrast, treatment with HNK obviously reversed the decrease of myocardial contraction, whereas there was no significant difference between the $\beta 1$-AR-ECII treatment and $\beta 1$-AR-ECII plus saline treatment groups (Figures 2(c) and $2(\mathrm{~d}))$.

To examine the cardiac systolic and diastolic function, we measured the parameters below 12 weeks after active immunization: left ventricular systolic pressure (LVSP), left ventricular end diastolic pressure (LVEDP), and maximal

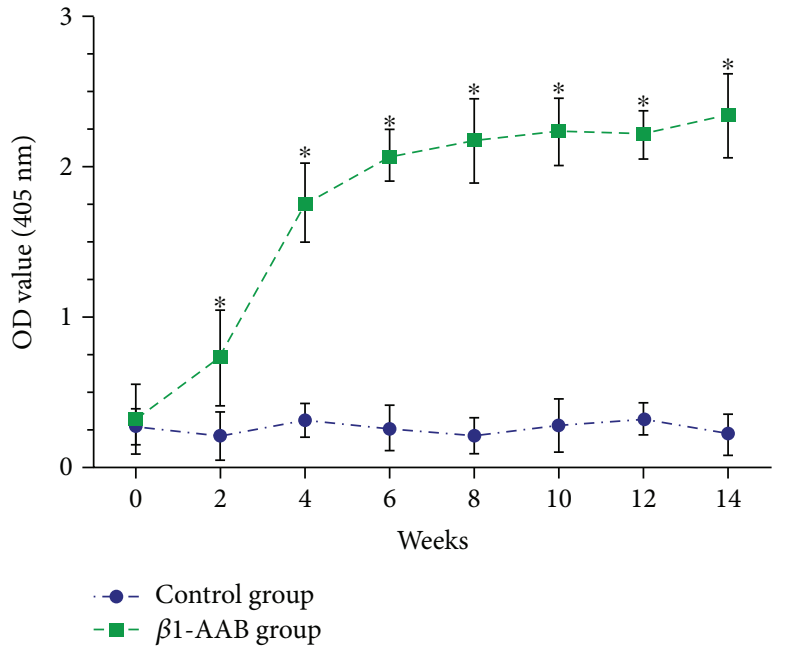

FIgURE 1: Immunization against $\beta 1$-AR-ECII induced a high-level accumulation of $\beta 1-\mathrm{AAB}$ in the blood. $\beta 1-\mathrm{AR}-\mathrm{ECII}$ ( $\beta 1-\mathrm{AAB}$ group) or saline (control group) were subcutaneously injected into adult rats. Antibody titer in the blood was defined by OD value. At the beginning, $\beta 1-\mathrm{AABs}$ in the two groups were similarly low. However, $\beta 1-\mathrm{AABs}$ were sharply increased in the first four weeks and maintained high in the $\beta 1$-AR-ECII treatment group. Data were expressed as mean $\pm \mathrm{SD}$ ( $n=12$ per group). ${ }^{*} P<0.05$.

positive and negative values of the instantaneous first derivative of left ventricular pressure $\left(+\mathrm{dP} / \mathrm{dt}_{\max }\right.$ and $\left.-\mathrm{dP} / \mathrm{dt}_{\max }\right)$. LVSP and $+\mathrm{dP} / \mathrm{dt}_{\max }$ represent systolic function. LVEDP and $-\mathrm{dP} / \mathrm{dt}_{\max }$ indicate diastolic function. Compared to the control group, the values of LVSP and $+\mathrm{dP}_{\mathrm{ddt}}$ max significantly reduced and the values of LVEDP and $-\mathrm{dP} / \mathrm{dt}_{\max }$ obviously increased in the $\beta 1$-AAB-positive group (Figure 3 ). $\mathrm{HNK}$ addition significantly reversed all these effects induced by $\beta 1-\mathrm{AAB}$, but there was no difference in the saline cotreatment group (Figure 3). These data indicate that the continuous immunization significantly inhibits cardiac ventricular contractility, but HNK-combined therapy obviously ameliorates the suppression of ventricular contractility and reverses cardiac dysfunction.

3.3. HNK Reduced Lactate Dehydrogenase (LDH) Activity and Attenuated Myocardial Degeneration. To detect the degree of cell injury, we measured the activity of LDH in each group. There was a significant elevation of $\mathrm{LDH}$ in the $\beta 1$ $\mathrm{AAB}$ group, and HNK addition remarkably decreased the level of LDH (Figure 4(a)). There was no significant difference between the $\beta 1-\mathrm{AAB}$ and $\beta 1-\mathrm{AAB}+$ vehicle groups (Figure $4(\mathrm{a})$ ). The results of $\mathrm{HE}$ staining in the $\beta 1$-AAB group two weeks after active immunization revealed that the myocardial cells underwent extensive pathological changes, including hypertrophy, disorganization, and more vacuoles with varying shapes (Figure 5(b)). These pathological changes were obviously ameliorated in the $\beta 1-\mathrm{AAB}+$ HNK group, but there was no significant change in the $\beta 1$ $\mathrm{AAB}+$ vehicle group (Figures 5(c) and 5(d)). These results indicate that HNK protects myocardial cells from breakage and degeneration directly or indirectly induced by highlevel $\beta 1-\mathrm{AAB}$. 


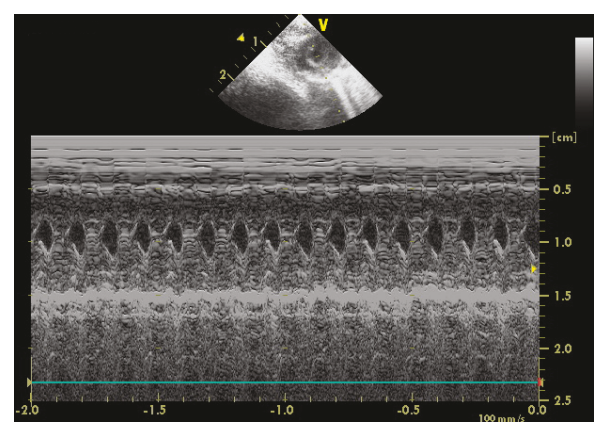

(a)

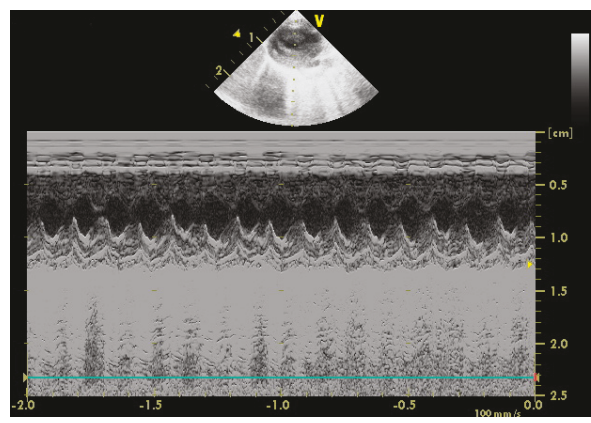

(c)

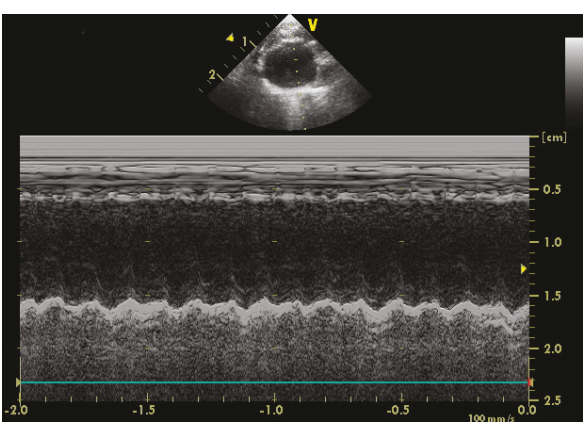

(b)

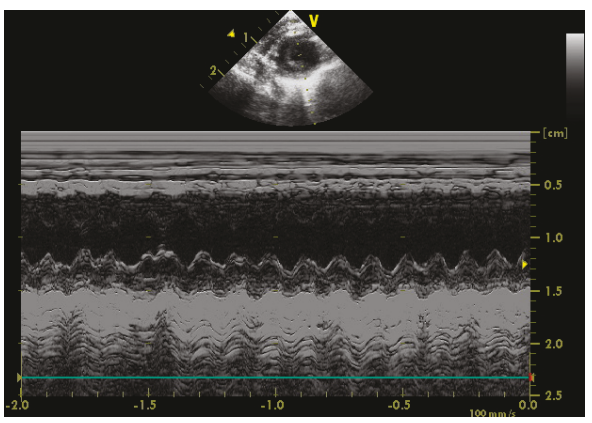

(d)

FIGURE 2: Honokiol ameliorated cardiac contractility suppressed by $\beta 1$-AAB. Representative M-mode echocardiograms were obtained from the rats at $12 \mathrm{~h}$ after each treatment. (a) Saline treatment (control group); (b) $\beta 1$-AR-ECII administration ( $\beta 1$-AAB group); (c) $\beta 1$-AR-ECII plus HNK administration ( $\beta 1-\mathrm{AAB}+\mathrm{HNK}$ group); and (d) $\beta 1$-AR-ECII plus saline administration $(\beta 1-\mathrm{AAB}+\mathrm{vehicle}$ group). HNK: honokiol.

3.4. HNK Activated Autophagy Inhibited by $\beta 1-A A B$. Microtubule-associated protein 1 light chain 3 I (LC3I) and LC3II are markers of autophagy. We demonstrated that the expression of LC3 in the $\beta 1$-AAB group was lower than that in the control group by immunofluorescence staining (Figure 4(b)). However, the staining intensity was stronger in the $\beta 1-\mathrm{AAB}$ $+\mathrm{HNK}$ group and it remained low in the $\beta 1-\mathrm{AAB}+$ vehicle group (Figure 4(b)). Notably, the Western blot data obtained after active immunization for four weeks were consistent with these results (Figures 6(a) and 6(b)). Beclin-1 is an important gene for autophagy. As p62 was degraded within the autolysosomes, it was usually used as an indicator of autophagic flux. To find out the effect of HNK on autophagy, the protein levels of LC3I and LC3II, Beclin-1, and p62 in the heart tissue of each group were detected. The protein levels of LC3I and LC3II and Beclin- 1 in the $\beta 1$-AAB group significantly reduced, while the level of p62 protein obviously increased (Figures 6(a)-6(d)). Compared to those of the $\beta 1-\mathrm{AAB}$ group, the protein levels of the $\beta 1-\mathrm{AAB}+\mathrm{HNK}$ group had the opposite change, while the addition of normal saline had no obvious effect on the protein level (Figure 6(a)6(d)). These data suggest that HNK is beneficial to the heart tissue by activating autophagy in a rat model of long-term maintenance of $\beta 1-\mathrm{AAB}$.

3.5. HNK Promoted the Activation of the AMP-Dependent Protein Kinase/Unc-51 Like Autophagy Activating Kinase Pathway. AMPK (AMP-dependent protein kinase) is a major energy-sensitive kinase regulating many cell processes, including macroautophagy. The phosphorylation of Unc51-like autophagy activating kinase (ULK) involves the formation of autophagosome [24]. To investigate whether the AMPK/ULK pathway was involved in the regulation of HNK, we used Western blot to measure the protein levels and phosphorylation patterns of AMPK and ULK four weeks after active immunization. We found that the levels of AMPK and ULK were not changed among the groups (Figures 7(a)-7(c)). However, the levels of phosphorylated AMPK and phosphorylated ULK were remarkably reduced in the $\beta 1-\mathrm{AAB}$ group, compared to the control group (Figures $7(\mathrm{a})-7(\mathrm{c})$ ). HNK cotreatment significantly increased these phosphorylated protein levels, but there was no obvious difference in the $\beta 1-\mathrm{AAB}+$ vehicle group (Figures $7(\mathrm{a})-7(\mathrm{c})$ ). These results indicate that the AMPK/ ULK pathway activated by HNK may be the intrinsic mechanism of increased autophagy.

\section{Discussion}

This study is to explore the effects of HNK on $\beta 1-\mathrm{AAB}$ positive heart diseases and the potential mechanisms. We established a rat model of long-term maintenance of $\beta 1$ $\mathrm{AAB}$ and demonstrated that the combination therapy of HNK prevented myocardial dysfunction and decreased $\mathrm{LDH}$ activity. We revealed that HNK was capable of attenuating myocardial degeneration. Additionally, we showed that HNK cotreatment promoted the activation of the AMPK/ ULK pathway and activated autophagy inhibited by high- 


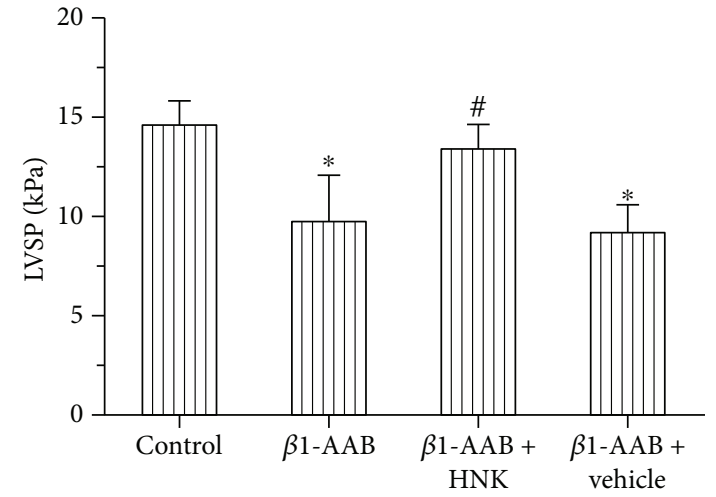

(a)

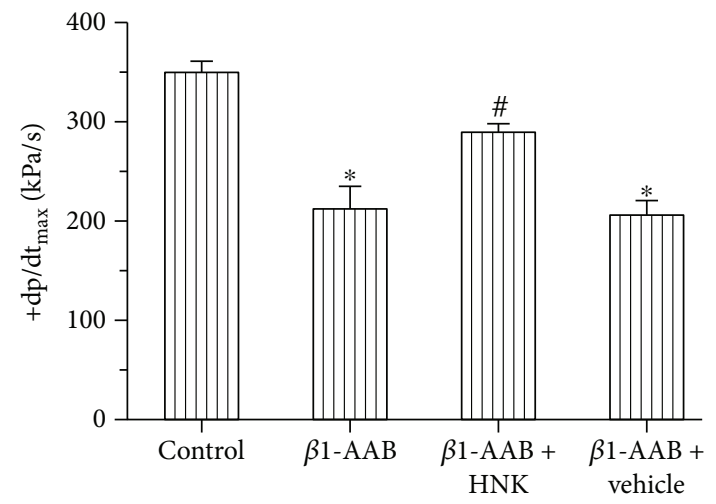

(c)

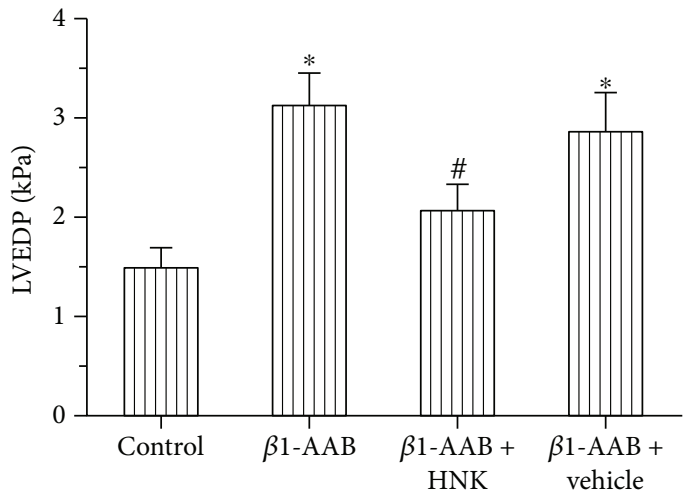

(b)

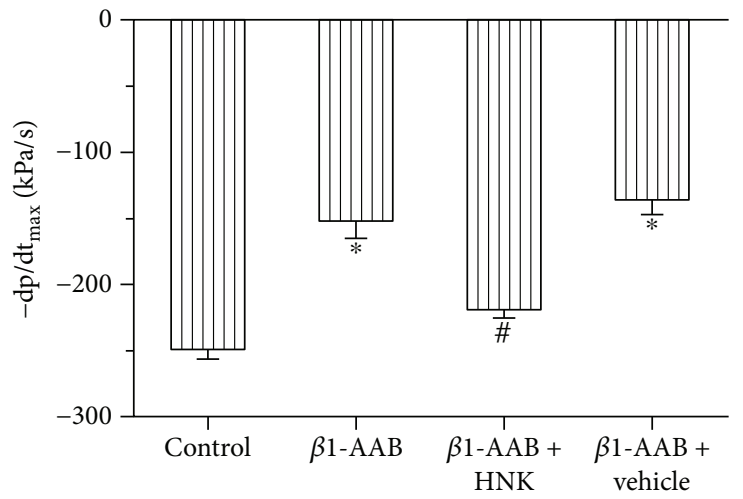

(d)

FIGURE 3: Honokiol protected cardiac systolic and diastolic function. Rats were randomly divided into the control group (saline treatment), $\beta 1$-AAB group ( $\beta 1$-AR-ECII treatment), $\beta 1$-AAB + HNK group ( $\beta 1$-AR-ECII plus HNK treatment), and $\beta 1$-AAB + vehicle group $(\beta 1-\mathrm{AR}-$ ECII plus saline treatment). LVSP (a), LVEDP (b), $+\mathrm{dP} / \mathrm{dt}_{\max }(\mathrm{c})$, and $-\mathrm{dP} / \mathrm{dt}_{\max }(\mathrm{d})$ were measured. In the $\beta 1-\mathrm{AAB}$ group, LVSP and $+\mathrm{dP} /$ $\mathrm{dt}_{\max }$ decreased and LVEDP and $-\mathrm{dP} / \mathrm{dt}_{\max }$ increased. These values changed in the opposite direction in the $\beta 1-\mathrm{AAB}+\mathrm{HNK}$ group, whereas there was no difference in the $\beta 1-\mathrm{AAB}+$ vehicle group. Data were expressed as mean $\pm \mathrm{SD}\left(n=12\right.$ per group). ${ }^{*} P<0.05$ relative to the control group. ${ }^{\#} P<0.05$ relative to the $\beta 1-\mathrm{AAB}$ group. HNK: honokiol.

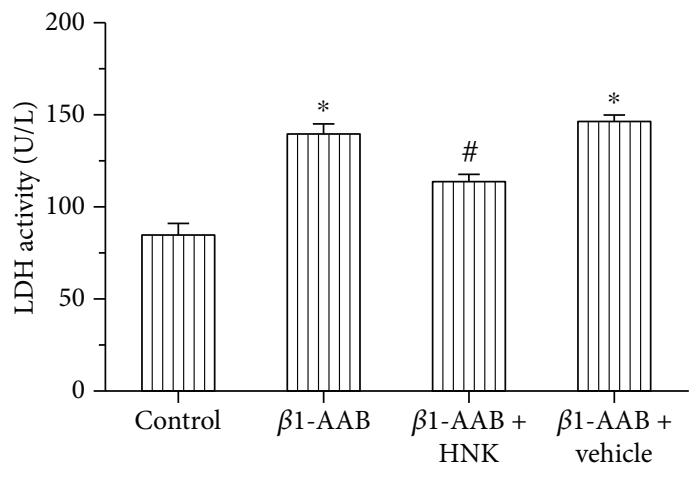

(a)
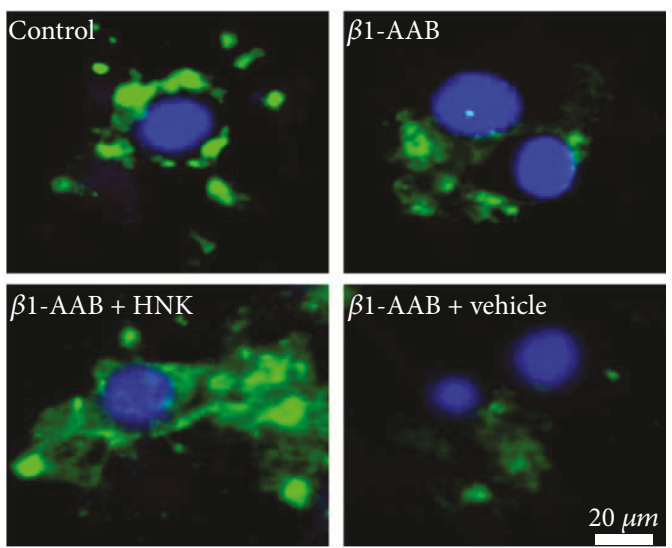

$\beta 1-\mathrm{AAB}+$ vehicle

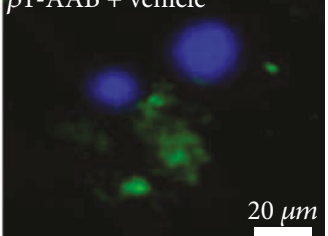

(b)

FIGURE 4: Honokiol reduced LDH activity and increased LC3 staining. H9c2 cells were randomly divided into the control group (saline treatment), $\beta 1-\mathrm{AAB}$ group ( $\beta 1$-AR-ECII treatment), $\beta 1-\mathrm{AAB}+\mathrm{HNK}$ group ( $\beta 1$-AR-ECII plus HNK treatment), and $\beta 1-\mathrm{AAB}+\mathrm{vehicle}$ group ( $\beta 1$-AR-ECII plus saline treatment). (a) The serum level of LDH in each group was determined. It increased in the $\beta 1-A A B$ group and significantly reduced in the $\beta 1-\mathrm{AAB}+\mathrm{HNK}$ group. (b) Representative immunofluorescent images of LC3 (green) and DAPI (blue) in each group. The staining intensity of LC3 reduced in the $\beta 1$-AAB group and increased in the $\beta 1-\mathrm{AAB}+\mathrm{HNK}$ group. Data were shown as mean \pm SD ( $n=6$ per group). ${ }^{*} P<0.05$ relative to the control group. ${ }^{\#} P<0.05$ relative to the $\beta 1-\mathrm{AAB}$ group. HNK: honokiol; LDH: lactate dehydrogenase. 


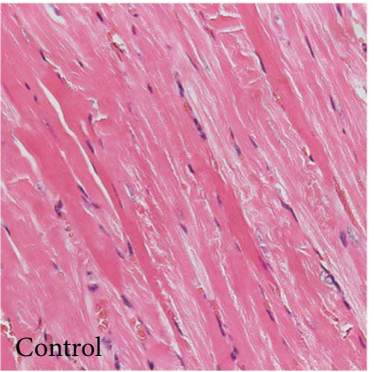

(a)

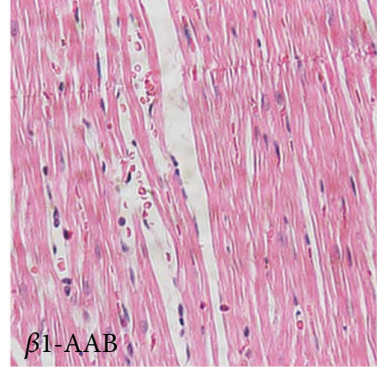

(b)

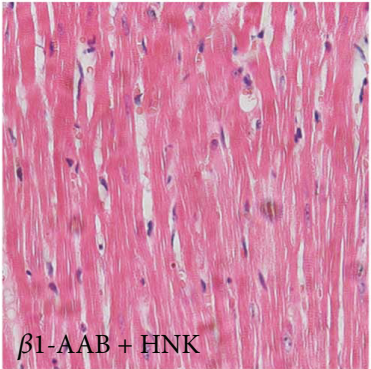

(c)

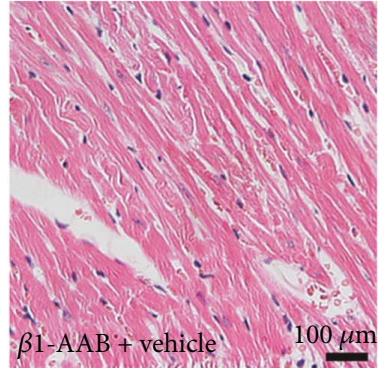

(d)

FIGURE 5: Honokiol attenuated the degeneration of cardiac tissue. (a), (b), (c), and (d) represented the HE staining images of cardiac tissue sections from the control, $\beta 1-\mathrm{AAB}, \beta 1-\mathrm{AAB}+\mathrm{HNK}$, and $\beta 1-\mathrm{AAB}+$ vehicle groups, respectively. (b) There was extensive myocardial degeneration in the $\beta 1-\mathrm{AAB}$ group. (c) A significant improvement of myocardial pathological features was observed in the $\beta 1-\mathrm{AAB}+$ HNK group. (d) There was no obvious amelioration of the pathological impairments in the $\beta 1-\mathrm{AAB}+$ vehicle group.

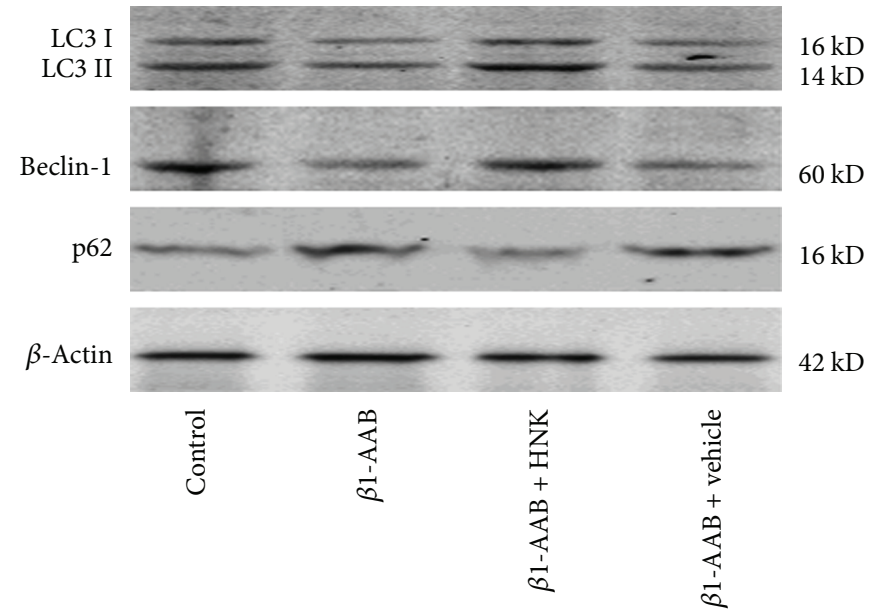

(a)

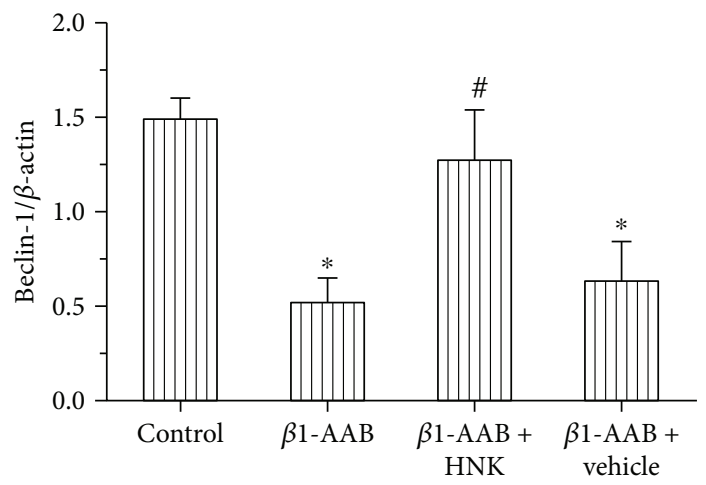

(c)

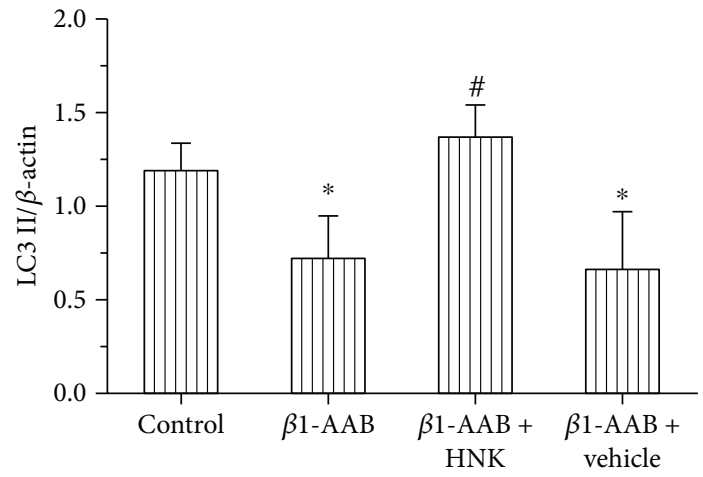

(b)

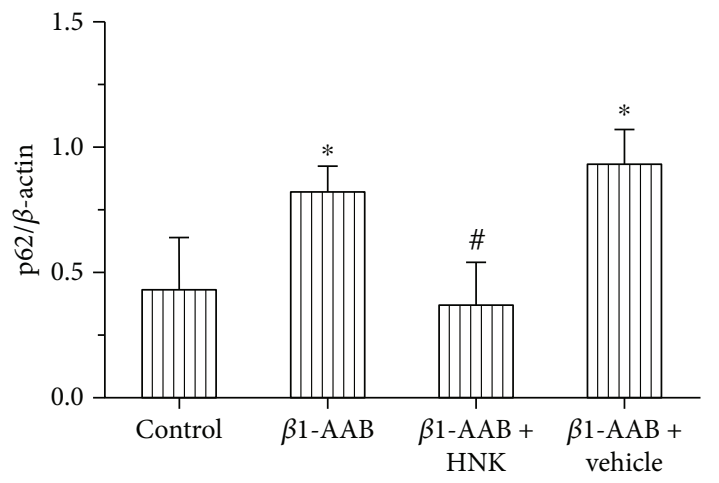

(d)

FIgURE 6: Honokiol activated autophagy in myocardial tissues after passive immunization. (a) Representative Western blot images of autophagic markers LC3I, LC3II, Beclin-1, and p62 in cardiac tissue lysates from each group. (b-d) Quantification and comparison of Western blot band values from (a). In the $\beta 1-\mathrm{AAB}$ group, LC3II and Beclin-1 decreased and p62 increased. These protein levels changed in the opposite direction in the $\beta 1-\mathrm{AAB}+\mathrm{HNK}$ group. Data were presented as mean $\pm \mathrm{SD}$ ( $n=12$ per group). ${ }^{*} P<0.05$ relative to the control group. ${ }^{\#} P<0.05$ relative to the $\beta 1-\mathrm{AAB}$ group. HNK: honokiol.

level of $\beta 1-\mathrm{AAB}$. To the best of our knowledge, this is the first time to demonstrate that $\mathrm{HNK}$ protects against $\beta 1-\mathrm{AAB}$ induced myocardial dysfunction. Furthermore, we have revealed that autophagy and the AMPK/ULK signaling pathway may underlie the benefits. $\beta 1-A A B$ was often detected in patients with various heart diseases [25]. It has been shown that $\beta 1-\mathrm{AAB}$ aggravates heart function [9]. HNK exerts protective functions in many vascular diseases [26]. Here, a rat model of long-term highlevel maintenance of $\beta 1-\mathrm{AAB}$ was established (Figure 1). 


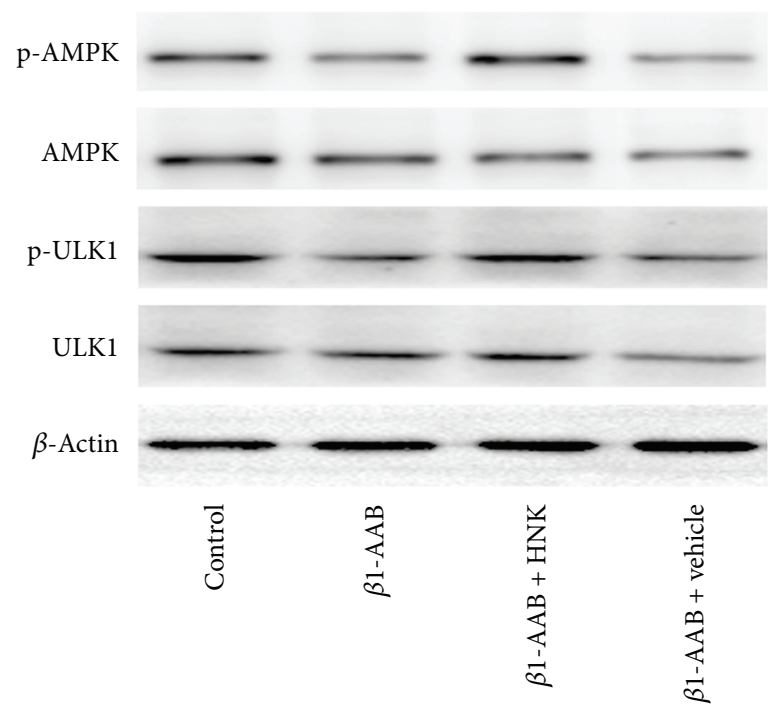

(a)

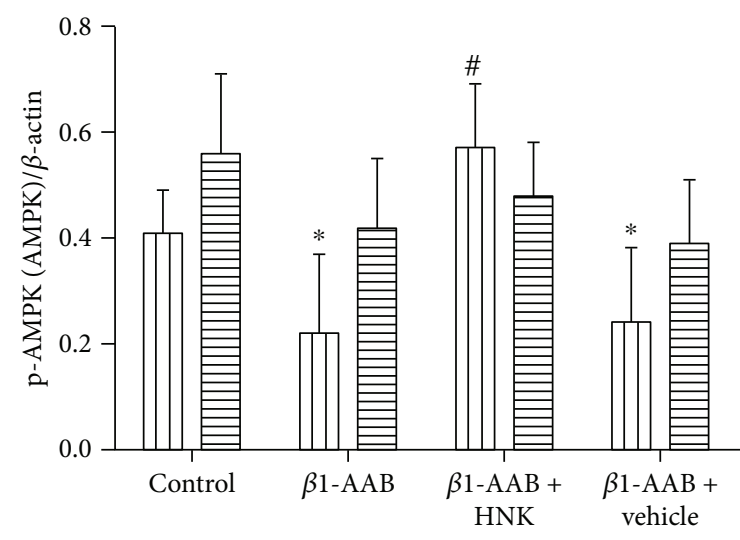

盂 $\mathrm{p}$-AMPK 冒 AMPK

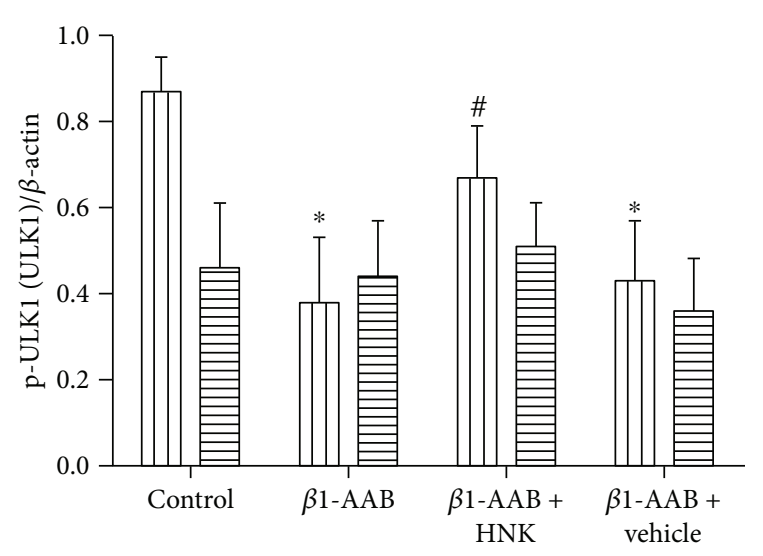

而 $\mathrm{p}$-ULK1 目 ULK1

(b)

(c)

FIgURE 7: Honokiol activated the AMPK/ULK1 pathway. (a) Representative Western blot images of p-AMPK (Thr172), AMPK, p-ULK1 (Ser555), and ULK1 in cardiac tissue lysates from each group. (b) Quantification and comparison of p-AMPK and AMPK band values from (a). (c) Quantification and comparison of p-ULK1 and ULK1 band values from (a). The phosphorylation of AMPK and ULK1 were inhibited by $\beta 1-\mathrm{AAB}$ and activated by HNK. Data were presented as mean $\pm \mathrm{SD}$ ( $n=12$ per group). ${ }^{*} P<0.05$ relative to the control group. ${ }^{\#} P<0.05$ relative to the $\beta 1-\mathrm{AAB}$ group. HNK: honokiol.

The continuous immunization caused significant suppression of cardiac ventricular contractility, and HNK cotreatment obviously ameliorated the adverse effect (Figures 2 and 3). These data indicate that HNK directly or indirectly regulates myocardial cell contractility. Cellular energy is critical for cell contractility [27]. The physiological maintenance of the respiratory chain needs the integrity of mitochondrial membrane [28]. $\beta 1$-AAB leads to the disruption of the electrochemical gradient $\Delta \Psi \mathrm{m}$ that reflects the integrity of the inner mitochondrial membrane [18]. HNK protects myocardial mitochondria from lipid peroxidation and decreases the myocardial infarct size caused by coronary occlusion [26]. In mice, HNK prevents cardiac hypertrophy by activating mitochondrial Sirt3 [29]. In our model, we propose that HNK may protect myocardial mitochondria from $\beta 1$-AABinduced impairments. It is reported that LDH leaks from damaged cells [30]. We demonstrated that HNK cotreatment inhibited the increase of LDH activity in the $\beta 1$-AAB-positive group (Figure 4(a)). Myocardial degeneration was also attenuated in the HNK cotreatment group (Figure 5). These results indicate that HNK may protect myocardial cells via mitochondrial regulation. A recent publication demonstrates that HNK protects the heart from Dox cardiotoxicity via improving mitochondrial function by not only repressing mitochondrial protein acetylation but also enhancing PPAR $\gamma$ activity in the heart [31].

Autophagy is a process of degradation of abnormal cellular components and necessary for maintaining cellular homeostasis [32]. Autophagy can be induced by mitochondrial impairments and inhibited by a $\beta$-adrenergic agonist [33]. LC3 is a component of the vacuoles during autophagy [34]. LC3I is a cytoplasmic, and LC3II is localized in the 
membrane of autophagosome. The increase of LC3II reflects the accumulation of autophagosomes, indicating the induction of autophagy [35]. Beclin-1 plays an important role in mediating the localization of autophagic proteins to preautophagosome [36]. P62 is degraded within the autolysosomes, the increase of which reflects the inhibition of autophagy [37]. Thus, LC3, Beclin-1, and p62 can be used as good indicators for autophagy analysis. The immunostaining and Western blot data showed that the levels of LC3II and Beclin-1 decreased and the level of p62 increased in the $\beta 1$ AAB-positive group. The addition of HNK significantly counteracted the effects (Figures 4(b) and 6). It is worth noting that HNK treatment promotes the recovery of systolic function and the inhibition of diastolic function (Figure 3). These data suggest that HNK protects myocardial functions by activating autophagy. Consistent with this, previous studies have shown that reduced autophagy contributes to heart disorders [38] and upregulated autophagy is remarkably beneficial for impaired cardiac function [39].

A complex signaling network regulates autophagy, most of which correlates with the AMP-activated protein kinase (AMPK)/ULK pathway [40]. It has been reported that Beclin-1 activity depends on the phosphorylation of AMPK [41]. Phosphorylated ULK1 is negatively regulated by mTOR that can be inhibited by AMPK [41]. HNK increased phosphorylated forms of both AMPK and ULK (Figure 7), indicating the underlying mechanism of increased autophagy. AMPK activation is mainly regulated by the phosphatases and kinases, and its distribution is tissue-specific. For instance, LKB1 kinase exists in the peripheral tissues, while CaMKK2 kinase is expressed in neurons [42]. Thus, it is likely that HNK activates AMPK via LKB1.

Combined with these data, we propose that HNK benefits cardiac tissue not by directly reducing the level of $\beta 1-\mathrm{AAB}$ in the sera.

\section{Conclusion}

In conclusion, this present study showed that $\mathrm{HNK}$ protected hearts from dysfunction in a rat model of $\beta 1$-AAB-positive, possibly by reducing the activity of $\mathrm{LDH}$, activating the AMPK/ULK pathway, and promoting autophagy. These results suggest that $\mathrm{HNK}$ may be a potentially therapeutic compound to cure $\beta 1$-AAB-positive heart diseases. These preliminary observations provide novel insights into the prevention of $\beta 1$-AAB-positive cadiovascular diseases. The detailed mechanisms for these additive effects remain unclear. It is worthwhile to investigate the effect of HNK on the mitochondria or energy metabolism in the future.

\section{Data Availability}

All data generated or analyzed in this study are included in this published article.

\section{Ethical Approval}

All the animal experiments were performed in accordance with the Guide for the Care and Use of Laboratory Animals published by the US National Institutes of Health (NIH publication no. 85-23, revised 1996), approved by the Institutional Animal Care and Use Committee of Qingdao University, and the Guide for the Care and Use of Laboratory Animals according to the regulation in the People's Republic of China. All animals were provided by Shanghai Experimental Animal Center, People's Republic of China. All surgery was performed under sodium pentobarbital anesthesia, and all efforts were made to minimize suffering.

\section{Conflicts of Interest}

The authors declare that they have no competing interests.

\section{Authors' Contributions}

Xi-qing Wei and Jun Yang designed the study. Xi-qing Wei, Guang-he Wei, Hong-sheng Zhang, Jin-guo Zhang, Yan-yan $\mathrm{Du}$, and Hong-yong Tan performed the experiments and collected the data. Xi-qing Wei, Guang-he Wei, Hong-sheng Zhang, and Hong-yong Tan analyzed and interpreted the experimental data. Xi-qing Wei and Jun Yang prepared the manuscript. Xi-qing Wei and Hong-sheng Zhang contributed equally to this work. All authors have consent for manuscript publication.

\section{Acknowledgments}

The authors are grateful to Hua Zhang for his technical assistance. This work was sponsored by the National Natural Science Foundation of China (812410006).

\section{References}

[1] J. Niu, A. Azfer, and P. E. Kolattukudy, "Protection against lipopolysaccharide-induced myocardial dysfunction in mice by cardiac-specific expression of soluble Fas," Journal of Molecular and Cellular Cardiology, vol. 44, no. 1, pp. 160169, 2008.

[2] M. M. Parker, K. E. McCarthy, F. P. Ognibene, and J. E. Parrillo, "Right ventricular dysfunction and dilatation, similar to left ventricular changes, characterize the cardiac depression of septic shock in humans," Chest, vol. 97, no. 1, pp. 126-131, 1990.

[3] Y. Wang, J. Mi, X.-Y. Shan, Q. J. Wang, and K.-Y. Ge, "Is China facing an obesity epidemic and the consequences? The trends in obesity and chronic disease in China," International Journal of Obesity, vol. 31, no. 1, pp. 177-188, 2007.

[4] P. Kaul, F. A. McAlister, J. A. Ezekowitz, V. K. Grover, and H. Quan, "Ethnic differences in 1-year mortality among patients hospitalised with heart failure," Heart, vol. 97, no. 13, pp. 1048-1053, 2011.

[5] H. Jiang and J. Ge, "Epidemiology and clinical management of cardiomyopathies and heart failure in China," Heart, vol. 95, no. 21, pp. 1727-1731, 2009.

[6] R. Jahns, V. Boivin, L. Hein et al., "Direct evidence for a $\beta_{1}$ adrenergic receptor-directed autoimmune attack as a cause of idiopathic dilated cardiomyopathy," The Journal of Clinical Investigation, vol. 113, no. 10, pp. 1419-1429, 2004.

[7] R. Jahns, V. Boivin, T. Krapf, G. Wallukat, F. Boege, and M. J. Lohse, "Modulation of beta ${ }_{1}$-adrenoceptor activity by domain- 
specific antibodies and heart failure-associated autoantibodies," Journal of the American College of Cardiology, vol. 36, no. 4, pp. 1280-1287, 2000.

[8] L. Sterin-Borda, G. Gorelik, M. Postan, S. Gonzalez Cappa, and E. Borda, "Alterations in cardiac beta-adrenergic receptors in chagasic mice and their association with circulating beta-adrenoceptor-related autoantibodies," Cardiovascular Research, vol. 41, no. 1, pp. 116-125, 1999.

[9] L. Zuo, H. Bao, J. Tian et al., "Long-term active immunization with a synthetic peptide corresponding to the second extracellular loop of $\beta 1$-adrenoceptor induces both morphological and functional cardiomyopathic changes in rats," International Journal of Cardiology, vol. 149, no. 1, pp. 89-94, 2011.

[10] T. Yamada, A. Matsumori, W. Z. Wang, N. Ohashi, K. Shiota, and S. Sasayama, "Apoptosis in congestive heart failure induced by viral myocarditis in mice," Heart and Vessels, vol. 14, no. 1, pp. 29-37, 1999.

[11] D. Jane-wit, C. Z. Altuntas, J. M. Johnson et al., “ $\beta_{1}$-adrenergic receptor autoantibodies mediate dilated cardiomyopathy by agonistically inducing cardiomyocyte apoptosis," Circulation, vol. 116, no. 4, pp. 399-410, 2007.

[12] L. Wang, H. Hao, J. Wang et al., "Decreased autophagy: a major factor for cardiomyocyte death induced by $\beta_{1}$-adrenoceptor autoantibodies," Cell Death \& Disease, vol. 6, no. 8, article e1862, 2015.

[13] L. E. Fried and J. L. Arbiser, "Honokiol, a multifunctional antiangiogenic and antitumor agent," Antioxidants \& Redox Signaling, vol. 11, no. 5, pp. 1139-1148, 2009.

[14] N. Matsui, K. Takahashi, M. Takeichi et al., "Magnolol and honokiol prevent learning and memory impairment and cholinergic deficit in SAMP8 mice," Brain Research, vol. 1305, pp. 108-117, 2009.

[15] Y. Wang, Z.-Z. Zhang, Y. Wu, J. Zhan, X.-H. He, and Y.L. Wang, "Honokiol protects rat hearts against myocardial ischemia reperfusion injury by reducing oxidative stress and inflammation," Experimental and Therapeutic Medicine, vol. 5, no. 1, pp. 315-319, 2013.

[16] S. K. Tsai, C. H. Huang, S. S. Huang, L. M. Hung, and C. Y. Hong, "Antiarrhythmic effect of magnolol and honokiol during acute phase of coronary occlusion in anesthetized rats: influence of L-NAME and aspirin," Pharmacology, vol. 59, no. 5, article 28324, pp. 227-233, 1999.

[17] K. T. Liou, S. M. Lin, S. S. Huang, C. L. Chih, and S. K. Tsai, "Honokiol ameliorates cerebral infarction from ischemiareperfusion injury in rats," Planta Medica, vol. 69, no. 2, pp. 130-134, 2003.

[18] L. Wang, K. Lu, H. Hao et al., "Decreased autophagy in rat heart induced by anti- $\beta 1$-adrenergic receptor autoantibodies contributes to the decline in mitochondrial membrane potential," PLoS One, vol. 8, no. 11, article e81296, 2013.

[19] V. B. Pillai, A. Kanwal, Y. H. Fang et al., "Honokiol, an activator of Sirtuin-3 (SIRT3) preserves mitochondria and protects the heart from doxorubicin-induced cardiomyopathy in mice," Oncotarget, vol. 8, no. 21, pp. 34082-34098, 2017.

[20] L. M. Semeniuk, A. J. Kryski, and D. L. Severson, "Echocardiographic assessment of cardiac function in diabetic $\mathrm{db} / \mathrm{db}$ and transgenic db/db-hGLUT4 mice," American Journal of Physiology. Heart and Circulatory Physiology, vol. 283, no. 3, pp. H976-H982, 2002.

[21] H. R. Liu, R. R. Zhao, X. Y. Jiao, Y. Y. Wang, and M. Fu, "Relationship of myocardial remodeling to the genesis of serum

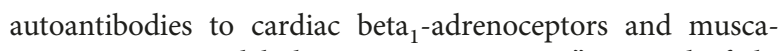
rinic type 2 acetylcholine receptors in rats," Journal of the American College of Cardiology, vol. 39, no. 11, pp. 18661873, 2002.

[22] E. Yamada, C. C. Bastie, H. Koga, Y. Wang, A. M. Cuervo, and J. E. Pessin, "Mouse skeletal muscle fiber-type-specific macroautophagy and muscle wasting are regulated by a Fyn/STAT3/ Vps34 signaling pathway," Cell Reports, vol. 1, no. 5, pp. 557$569,2012$.

[23] Y. Nagatomo, D. Li, J. Kirsop, A. Borowski, A. Thakur, and W. H. W. Tang, "Autoantibodies specifically against $\beta 1$ adrenergic receptors and adverse clinical outcome in patients with chronic systolic heart failure in the $\beta$-blocker era: the importance of immunoglobulin G3 subclass," Journal of Cardiac Failure, vol. 22, no. 6, pp. 417-422, 2016.

[24] K. R. Parzych and D. J. Klionsky, "An overview of autophagy: morphology, mechanism, and regulation," Antioxidants \& Redox Signaling, vol. 20, no. 3, pp. 460-473, 2014.

[25] Y. Magnusson, G. Wallukat, F. Waagstein, A. Hjalmarson, and J. Hoebeke, "Autoimmunity in idiopathic dilated cardiomyopathy. Characterization of antibodies against the beta 1adrenoceptor with positive chronotropic effect," Circulation, vol. 89, no. 6, pp. 2760-2767, 1994.

[26] E. Jing, B. Emanuelli, M. D. Hirschey et al., "Sirtuin-3 (Sirt3) regulates skeletal muscle metabolism and insulin signaling via altered mitochondrial oxidation and reactive oxygen species production," Proceedings of the National Academy of Sciences of the United States of America, vol. 108, no. 35, pp. 14608-14613, 2011.

[27] A. Kaasik, F. Joubert, R. Ventura-Clapier, and V. Veksler, "A novel mechanism of regulation of cardiac contractility by mitochondrial functional state," The FASEB Journal, vol. 18, no. 11, pp. 1219-1227, 2004.

[28] G. Kroemer, N. Zamzami, and S. A. Susin, "Mitochondrial control of apoptosis," Immunology Today, vol. 18, no. 1, pp. 44-51, 1997.

[29] V. B. Pillai, S. Samant, N. R. Sundaresan et al., "Honokiol blocks and reverses cardiac hypertrophy in mice by activating mitochondrial Sirt3," Nature Communications, vol. 6, no. 1, p. 6656, 2015.

[30] H. Y. Sun, N. P. Wang, F. Kerendi et al., "Hypoxic postconditioning reduces cardiomyocyte loss by inhibiting ROS generation and intracellular $\mathrm{Ca}^{2+}$ overload," American Journal of Physiology. Heart and Circulatory Physiology, vol. 288, no. 4, pp. H1900-H1908, 2005.

[31] L. Huang, K. Zhang, Y. Guo et al., "Honokiol protects against doxorubicin cardiotoxicity via improving mitochondrial function in mouse hearts," Scientific Reports, vol. 7, no. 1, article 11989, 2017.

[32] D. J. Klionsky and S. D. Emr, “Autophagy as a regulated pathway of cellular degradation," Science, vol. 290, no. 5497, pp. 1717-1721, 2000.

[33] H. Sandoval, P. Thiagarajan, S. K. Dasgupta et al., "Essential role for Nix in autophagic maturation of erythroid cells," Nature, vol. 454, no. 7201, pp. 232-235, 2008.

[34] N. Mizushima, "Methods for monitoring autophagy," The International Journal of Biochemistry \& Cell Biology, vol. 36, no. 12, pp. 2491-2502, 2004.

[35] T. Shintani and D. J. Klionsky, "Autophagy in health and disease: a double-edged sword," Science, vol. 306, no. 5698, pp. $990-995,2004$. 
[36] Y. Takahashi, D. Coppola, N. Matsushita et al., "Bif-1 interacts with Beclin 1 through UVRAG and regulates autophagy and tumorigenesis," Nature Cell Biology, vol. 9, no. 10, pp. 11421151, 2007.

[37] M. Komatsu, S. Waguri, M. Koike et al., "Homeostatic levels of p62 control cytoplasmic inclusion body formation in autophagy-deficient mice," Cell, vol. 131, no. 6, pp. 11491163, 2007.

[38] A. Sun, Y. Cheng, Y. Zhang et al., "Aldehyde dehydrogenase 2 ameliorates doxorubicin-induced myocardial dysfunction through detoxification of 4-HNE and suppression of autophagy," Journal of Molecular and Cellular Cardiology, vol. 71, pp. 92-104, 2014.

[39] M. S. Bhuiyan, J. S. Pattison, H. Osinska et al., "Enhanced autophagy ameliorates cardiac proteinopathy," The Journal of Clinical Investigation, vol. 123, no. 12, pp. 5284-5297, 2013.

[40] J. Wang, Q. Qi, Z. Feng et al., "Berberine induces autophagy in glioblastoma by targeting the AMPK/mTOR/ULK1-pathway," Oncotarget, vol. 7, no. 41, pp. 66944-66958, 2016.

[41] E. Yamada, S. Okada, C. C. Bastie et al., "Fyn phosphorylates AMPK to inhibit AMPK activity and AMP-dependent activation of autophagy," Oncotarget, vol. 7, no. 46, pp. 7461274629, 2016.

[42] T. E. Jensen, A. J. Rose, S. B. Jørgensen et al., "Possible CaMKK-dependent regulation of AMPK phosphorylation and glucose uptake at the onset of mild tetanic skeletal muscle contraction," American Journal of Physiology. Endocrinology and Metabolism, vol. 292, no. 5, pp. E1308-E1317, 2007. 


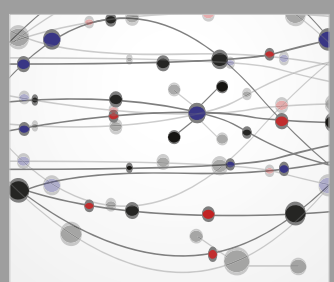

The Scientific World Journal
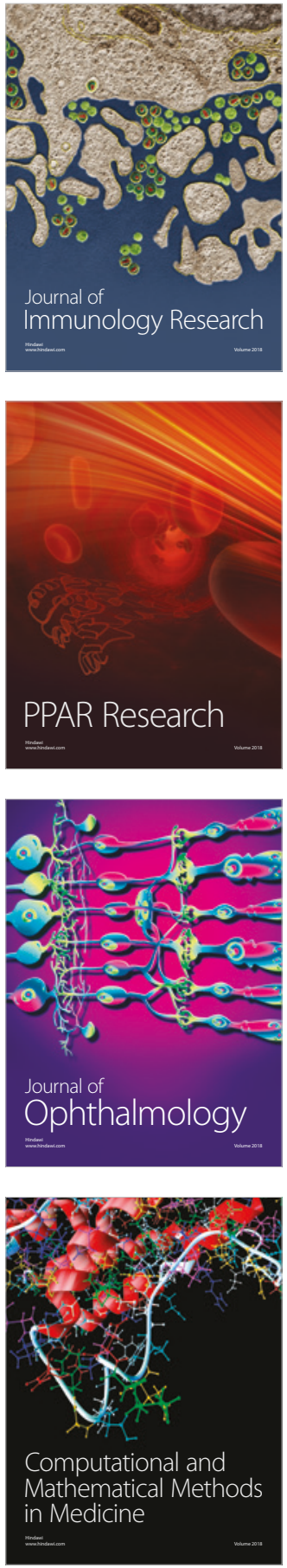

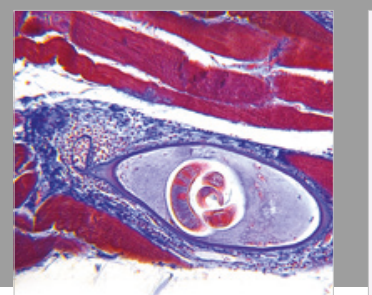

Gastroenterology Research and Practice

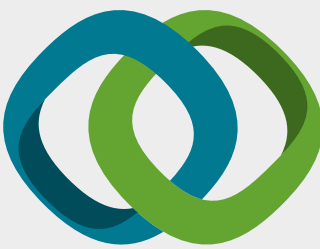

\section{Hindawi}

Submit your manuscripts at

www.hindawi.com
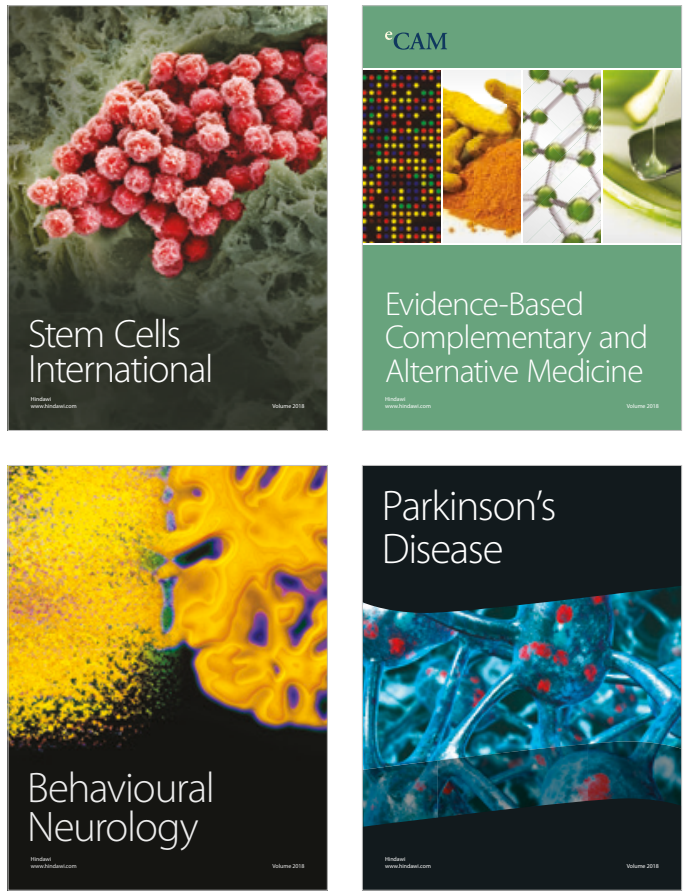

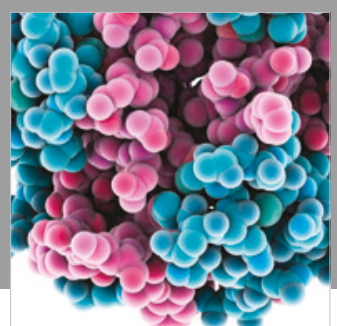

ournal of

Diabetes Research

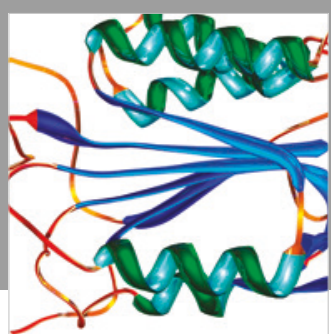

Disease Markers
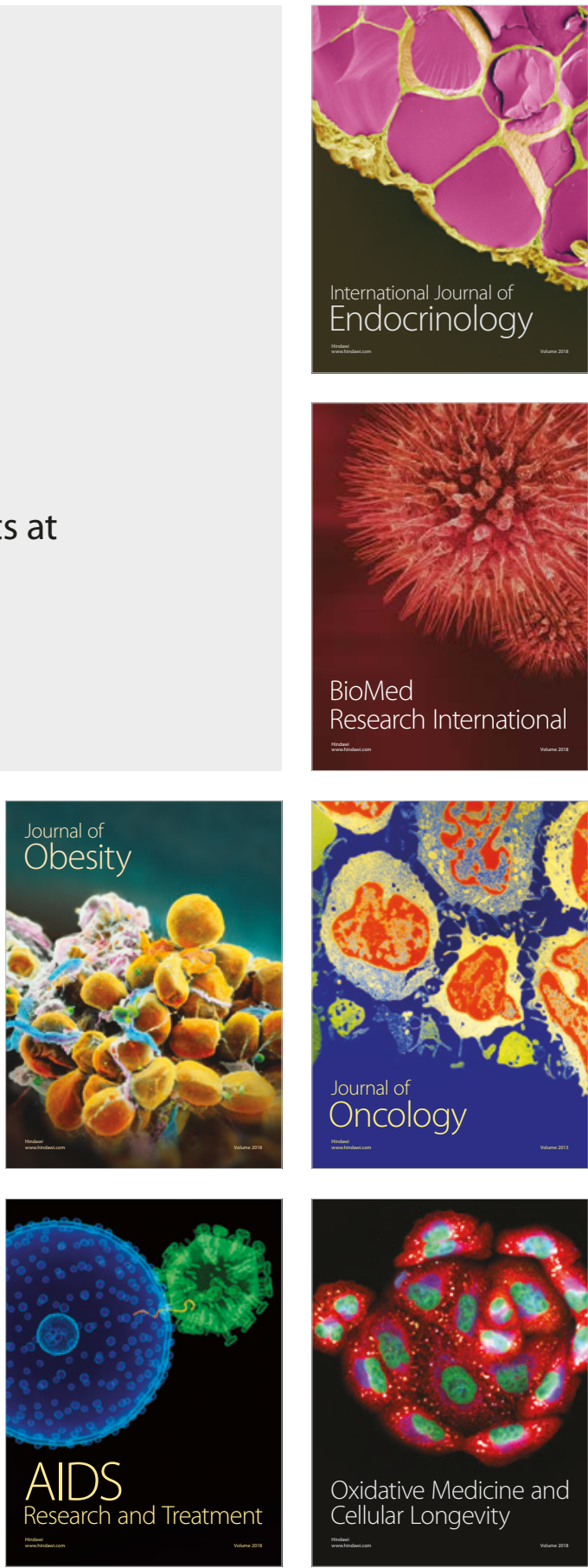